Karl-Franzens Universität Graz

Technische Universität Graz

Medizinische Universität Graz

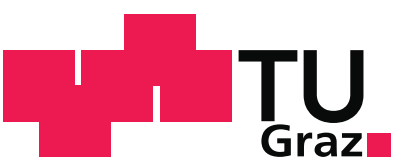

\title{
Application of optimal control to the cardiac defibrillation problem using a physiological model of cellular dynamics.
}
C. Nagaiah
K. Kunisch
G. Plank

SFB-Report No. 2015-018

January 2015

This article is a preprint. It was published in Applied Numerical Mathematics by

Elsevier. The version of record is available at http://dx.doi.org/doi:10.1016/j.apnum.2015.01.006.

Supported by the Austrian Science Fund (FWF)

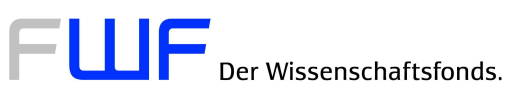

SFB sponsors:

- Austrian Science Fund (FWF)

- University of Graz

- Graz University of Technology

- Medical University of Graz

- Government of Styria

- City of Graz 


\title{
Application of optimal control to the cardiac defibrillation problem using a physiological model of cellular dynamics
}

\author{
Nagaiah Chamakuri ${ }^{\mathrm{a}, *}$, Karl Kunisch ${ }^{\mathrm{a}, \mathrm{b}}$, Gernot Plank ${ }^{\mathrm{c}}$ \\ ${ }^{a}$ Radon Institute for Computational and Applied Mathematics, Altenbergerstr. 69, Linz, \\ A-4040 Austria. \\ ${ }^{b}$ Institute of Mathematics and Scientific Computing, University of Graz, Heinrichstr. 36, \\ Graz, A-8010 Austria. \\ ${ }^{c}$ Institute of Biophysics, Medical University of Graz, Harrachgasse 21, Graz, A-8010 Austria.
}

\begin{abstract}
Optimal control techniques are investigated with the goal of terminating reentry waves in cardiac tissue models. In this computational study the Luo-Rudy phase I ventricular action potential model is adopted which accounts for more biophysical details of cellular dynamics as compared to previously used phenomenological models. The parabolic and ordinary differential equations are solved as a coupled system and an AMG preconditioner is used to solve the discretized elliptic equation The numerical results demonstrate that defibrillation is possible by delivering a single strong shock. The optimal control approach also leads to successful defibrillation and demands less total current. The present study motivates us to further investigate optimal control techniques on realistic geometries by incorporating the structural heterogeneity in the cardiac tissue.
\end{abstract}

Keywords: bidomain model, Luo-Rudy model, defibrillation, Neumann boundary stimulation, optimal control, parallel FEM.

\section{Introduction}

Cardiac rhythm disorders are among the leading causes of death in the industrialized world. The heart is an electrically controlled mechanical pump whose vital function is to drive blood through the circulatory system to supply organs with oxygen and metabolites. Under healthy conditions the heart fulfills this duty with remarkable efficiency by synchronizing mechanical contraction via fast, highly organized electrical activation of the ventricles, i.e. the main pumping chambers of the heart. Under pathological conditions electrical activation patterns becomes less regular and may, ultimately, transition to highly disorganized

\footnotetext{
* Corresponding author

Email addresses: nagaiah.chamakuri@ricam. oeaw.ac.at (Nagaiah Chamakuri), karl.kunisch@uni-graz.at (Karl Kunisch), gernot.plank@medunigraz.at (Gernot Plank)
} 
reentrant activation patterns, referred to as ventricular fibrillation. Under such conditions mechanical pumping function is severely impaired and sudden cardiac death ensues within minutes without appropriate interventions. The only reliable therapy to restore a normal heart rhythm is the timely application of a strong electrical shock, a procedure referred to as electrical defibrillation therapy, which terminates reentrant activations.

In this study, we explore the use of electrical defibrillation shocks to terminate reentrant activation and the implications of employing optimal control techniques to obtain optimal shock waveforms. In particular, we focus on the termination of reentrant spiral waves, a scenario which would be classified as monomorphic ventricular tachycardia in a clinical context. While non-lethal, spiral waves are often a precursor of more severe rhythm disturbances such as ventricular fibrillation.

In literature, one of the most accurate models of cardiac bioelectricity is the bidomain model, which describes both the extracellular and the intracellular potentials. Mathematically, the bidomain model consists of partial differential equations coupled with ordinary differential equations which model the associated ionic currents traversing the membranes of cardiac cells [14, 22, 29]. The bidomain model can be cast into an elliptic partial differential equation (PDE) that links the distribution of the transmembrane voltage, within the tissue to the extracellular potential, and a parabolic PDE that describes the cellular activation and recovery processes and the diffusive effect onto the adjacent tissue. Ordinary differential equations (ODEs) are employed to model the dynamical behavior of electricity in the myocardial cells. The dimension of the system of ODEs depends on the chosen membrane model. The present work focuses on the Luo-Rudy phase-I [17] ionic model which is a widely used computational model for the guinea pig ventricular action potentials. This model extends the BeelerReuter model [2] to enhance the representation of depolarization and repolarization phases and their interaction, its state space of the model consists of 6 gating variables and calcium concentration.

The optimal control approach to defibrillation seeks to determine an applied electrical field in such a way that a given design objective, which is, in our case, the restoration of a tissue state where the propagation of spiral waves is terminated, is minimized. For this purpose most of the tissue is driven to an excited state, such that the excitable gap, i.e. the portion of the tissue sufficiently close to the resting state to be excitable, is small, thus minimizing the space for the movement of reentrant waves. Achieving these objectives is challenging since, on biophysical grounds, defibrillation shocks always induced changes in polarization of both polarities [25, 21]. That is, the application of shocks always induces hyperpolarization in different regions of the heart, even regions which were refractory prior to shock administration may recover excitability, a phenomenon referred to as shock-induced deexcitation [11]. The optimal extracellular current density, injected through a set of electrodes, establishes an extracellular potential distribution which can dampen the voltage gradients in the tissue during the post 
shock simulations.

The optimal control approach is based on minimizing a properly chosen cost functional

$$
J\left(v, I_{e}\right)
$$

depending on the extracellular current $I_{e}$ as input and on the transmembrane potential $v$ as one of the state variables. In previous work $[7,8,6]$ the controller action representing the current delivered by electrodes was modeled as distributed force. Recently, we modeled the injected current via Neumann boundary conditions in the bidomain equations [9] using the simplified FitzHugh-Nagumo ionic model [23].

Turning to numerical aspects, it is well known that solving the bidomain equations is an inherently expensive procedure. The fast upstroke of the cardiac action potential translates into steep wave fronts in space, thus necessitating very fine spatio-temporal discretizations $[32,18]$. In our study, we have chosen the piecewise linear finite element method for the spatial discretization and higher order linearly implicit Runge-Kutta time stepping methods for the temporal discretization. There are numerous efforts to solve the linear algebraic system of the bidomain models efficiently [20]. Here we have chosen the algebraic multigrid method $[27,15,3]$ as preconditioner to solve the elliptic system, since it has been demonstrated that AMG is a highly efficient preconditioner for this particular problem [20]. It is well know that the building the matrix hierarchy on coarser level demands a lot of computational time for AMG. In this regard, we built the matrix hierarchy at the first iteration of the temporal loop and reused it for the subsequent time steps in the AMG solver. To solve the coupled parabolic and ODEs we used the ROS3PL [16] method which has 4 internal stages and is third order accurate. We stress that the system matrix is same during all internal stages at each time step. Therefore, we use the standard BiCGSTAB method with ILU preconditioner at each stage. Here as well, we compute the LU decomposition only once at each time step and reuse it while solving each internal stage of the ROS3PL method. In this way we could save the computational time for the matrix decomposition at each internal stage of the Rosenbrock method.

The organization of the remaining article is as follows: The bidomain model equations along with the Luo-Rudy ionic model is explained in the next section. The optimal control formulation and the derivation of the optimality system is given in Section 3. In Section 4, the numerical discretization of the primal and dual equations to solve the optimality conditions is explained. Furthermore, the solution procedure to solve the decoupled elliptic and parabolic part, as well the optimization algorithm is explained. Numerical results showing optimal defibrillation are given in Section 5. A short Conclusion section ends the paper. 


\section{Bidomain model equations}

We denote by $\Omega \subset \mathbb{R}^{2}$ a bounded connected domain with Lipschitz continuous boundary $\partial \Omega$. For brevity, the space-time domain and its lateral boundary are denoted by $Q=\Omega \times(0, T]$ and $\Sigma=\partial \Omega \times(0, T]$, respectively. The dynamical behavior of the cardiac tissue is described as a two-phase medium, one phase represents the intracellular space, the other one the extracellular space. The two phases are connected by a network of resistors, representing the ion channels, and capacitors, representing a capacitative current driven across the membranes by the potential difference, see e.g. [14, 22, 29]. Mathematically, the complete description of cardiac electricity is given by the following well known bidomain equations which consist of a linear elliptic partial differential equation and a nonlinear parabolic partial differential equation of reaction-diffusion type, where the reaction term is described by a set of ordinary differential equations:

$$
\begin{aligned}
0 & =\nabla \cdot\left(\bar{\sigma}_{i}+\bar{\sigma}_{e}\right) \nabla u+\nabla \cdot \bar{\sigma}_{i} \nabla v \text { in } Q \\
\frac{\partial v}{\partial t} & =\nabla \cdot \bar{\sigma}_{i} \nabla v+\nabla \cdot \bar{\sigma}_{i} \nabla u-I_{\text {ion }}(v, \overline{\mathbf{w}})+I_{\text {stim }} \text { in } Q \\
\frac{\partial \overline{\mathbf{w}}}{\partial t} & =G(v, \overline{\mathbf{w}}) \text { in } Q .
\end{aligned}
$$

Here $v: Q \rightarrow \mathbb{R}$ is the transmembrane voltage, $\overline{\mathbf{w}}: Q \rightarrow \mathbb{R}^{n}$ represents the $n$ ionic current variables, $\bar{\sigma}_{e}, \bar{\sigma}_{i}: \Omega \rightarrow \mathbb{R}^{d \times d}$ are respectively the extra and intracellular conductivity tensors, $I_{i o n}$ is the ionic current, and $I_{\text {stim }}$ is the stimulus current which is key to exciting the system. Eq. (3) is a parabolic equation and Eq. (4) is a set of ordinary differential equations which can be solved independently for each node. The transmembrane potential is defined by $v=u_{i}-u$, where $u_{i}$ and $u: Q \rightarrow \mathbb{R}$ are the intracellular and extracellular potentials. Moreover, the dimension of the ODE system is a consequence of the ionic model.

In our computations we considered the Luo-Rudy phase-I model (LR1) [17] which extends the Beeler-Reuter model to enhance the representation of depolarization and repolarization phases and their interaction. The time course of the action potential is governed by a set of ionic currents

$$
I_{i o n}=I_{N a}+I_{s i}+I_{K}+I_{K 1}+I_{K p}+I_{b} .
$$

which are fast sodium current $\left(I_{N a}\right)$, slow inward calcium current $\left(I_{s i}\right)$, time dependent potassium current $\left(I_{K}\right)$, time independent potassium current $\left(I_{K 1}\right)$, plateau potassium current $\left(I_{K p}\right)$ and background current $\left(I_{b}\right)$. The time dependent currents, $I_{N a}, I_{s i}$ and $I_{K}$, depend on six activation and inactivation gates $m$, $h, j, d, f, X$, which are governed by ordinary differential equations of the form

$$
\frac{d g}{d t}=\alpha_{g}(v)(1-g)-\beta_{g}(v) g, \quad \text { where } g=m, h, j, d, f, X .
$$


The $\alpha$ 's and $\beta$ 's, taking values between 0 and 1, are given by explicit formulas as functions of transmembrane voltage $v$. For further discussion on formulation of those functions and the parameters used in our computations we refer to the original paper of Luo-Rudy phase-I (LR1) model [17]. The existence and uniqueness for the LR1 model and more general of the classical Hodgkin-Huxley model can be found in Veneroni [31].

Cardiac tissue is known to be an anisotropic material, its eigenaxes are a function of space. The principal axis is aligned with the prevailing orientation of myocytes and is referred to as "fiber orientation". Spatial variation of fiber orientation in space is referred to as fiber curvature. In the transmural direction, the helix angle of fibers changes linearly with space, which is referred to as fiber rotation. Both fiber rotation and curvature are known to be important for mediating shock-induced changes in membrane polarization in the far-field, i.e. several space constants away from tissue surface and electrode location. In absence of spatial heterogeneity in fiber orientation tissue in the far-field such as in the depth of the myocardial walls would remain unaffected by an applied electric field, thus preventing electrical defibrillation shocks to terminate reentrant activity there[26, 28]. In our computations, the conductivity tensors are used in the following form,

$$
\sigma_{c}=\left(\begin{array}{cc}
\sigma_{c l} & 0 \\
0 & \sigma_{c t}
\end{array}\right), \quad \text { where } c=i, e,
$$

where $\sigma_{c l}$ and $\sigma_{c t}$ are longitudinal and transverse conductivities, respectively. Moreover, in our numerical simulations the spatial heterogeneity of the tensor is derived from a short axis histological image of a ventricular wall. The procedure for estimating fiber orientation from images is explained later in Section 5.

The initial and boundary conditions are prescribed as

$$
\begin{aligned}
\eta \cdot\left(\sigma_{i} \nabla v+\sigma_{i} \nabla u\right) & =0 \quad \text { on } \Sigma \\
\eta \cdot \sigma_{e} \nabla u & =I_{e}(t) \quad \text { on } \quad \partial \Omega_{12} \times(0, T] \\
\eta \cdot \sigma_{e} \nabla u & =0 \quad \text { on } \partial \Omega_{3} \times(0, T] \\
v(x, 0)=v_{0}, & \overline{\mathbf{w}}(x, 0)=\overline{\mathbf{w}}_{0} \quad \text { on } \Omega,
\end{aligned}
$$

where $\eta$ denotes the outwards normal to the boundary of $\Omega$. Here $I_{e}(t)$ is the extracellular current density stimulus which acts as control along the boundary $\partial \Omega_{12}=\partial \Omega_{1} \cup \partial \Omega_{2}$, where $\partial \Omega_{i}, i=1,2,3$ are mutually disjoint and satisfy $\partial \Omega_{1} \cup$ $\partial \Omega_{2} \cup \partial \Omega_{3}=\partial \Omega$. For compatibility reasons it is assumed throughout that

$$
\int_{s} I_{e}(t, \cdot) d s=0, \text { where } s=\partial \Omega_{12}
$$

for almost every $t \in(0, T)$. In the numerical experiments $I_{e}$ will be only temporally dependent and will be of the form

$$
I_{e}(t, \cdot)=\hat{I}_{e}(t)\left(\chi_{\partial \Omega_{1}}-\chi_{\partial \Omega_{2}}\right),
$$


where $\chi_{\partial \Omega_{i}}$ is the characteristic function of the set $\partial \Omega_{i}, i=1,2$. Then condition (11) is satisfied if $\left|\partial \Omega_{1}\right|=\left|\partial \Omega_{2}\right|$. The support regions $\partial \Omega_{1}$ and $\partial \Omega_{2}$ can be considered to represent an anode and a cathode respectively.

\section{Optimal control}

We explain the optimal control formulation and the derivation of optimality conditions in this section. First, we describe the cost function which is used in our computations that determine the controlled trajectory of the transmembrane voltage to a given desired state $v_{d}$ and accomplish the characteristic pattern of polarization in the cardiac tissue. The final pattern of polarization may lead to successful defibrillation during the post shock simulations. Here we consider that the extracellular current $I_{e}(t)$ serves as a control in the cost functional $J\left(v, I_{e}\right)$ and has an averse effect on the tissue. In computations, the following cost functional of tracking type is considered.

$$
\left\{\begin{array}{l}
\min J\left(v, I_{e}\right)=\frac{1}{2} \int_{0}^{T}\left(\alpha_{1} \int_{\Omega_{o b s}}\left|v-v_{d}\right|^{2} d x+\alpha_{2} \int_{\partial \Omega_{12}} I_{e}(t)^{2} d s\right) d t \\
\text { subject to (2)-(4),(7)-(10) and } I_{e} \in U
\end{array}\right.
$$

where $\alpha_{1}>0, \alpha_{2}>0$ is the regularization parameter for the control cost, $\Omega_{o b s} \subset \Omega$ is the observation domain, $v_{d} \in L^{2}\left(0, T ; L^{2}\left(\Omega_{o b s}\right)\right)$, and

$$
\begin{array}{r}
U=\left\{I_{e}-\frac{1}{\left|\partial \Omega_{12}\right|} \int_{\partial \Omega_{12}} I_{e} d s: I_{e} \in L^{2}\left(0, T ; L^{2}\left(\partial \Omega_{12}\right)\right),\left|I_{e}(t, x)\right| \leq R\right. \\
\text { for a.e. } \left.(t, x) \in(0, T) \times \partial \Omega_{12}\right\},
\end{array}
$$

where $\partial \Omega_{12}=\partial \Omega_{1} \cup \partial \Omega_{2}$. The set of admissible controls $U$ is a closed, convex and weakly* sequentially compact subset of $L^{\infty}\left(0, T ; L^{2}\left(\partial \Omega_{12}\right)\right)$. For computational purposes the first order necessary conditions are of paramount importance. We give a formal derivation of these conditions here. To compute the gradient of reduced cost functional we introduce the following Lagrangian which is related to the optimal control problem for the current problem.

$$
\begin{aligned}
\mathcal{L}\left(u, v, f, s, I_{e}, p, q, r, o\right)=J\left(v, I_{e}\right) & \\
& +\int_{0}^{T} \int_{\Omega}\left(\nabla \cdot\left(\bar{\sigma}_{i}+\bar{\sigma}_{e}\right) \nabla u+\nabla \cdot \bar{\sigma}_{i} \nabla v\right) p \mathrm{~d} \Omega \mathrm{d} t \\
& +\int_{0}^{T} \int_{\Omega}\left(\nabla \cdot \bar{\sigma}_{i} \nabla v+\nabla \cdot \bar{\sigma}_{i} \nabla u-\frac{\partial v}{\partial t}-I_{i o n}(v, \overline{\mathbf{w}})\right) q \mathrm{~d} \Omega \mathrm{d} t \\
& +\int_{0}^{T} \int_{\Omega}\left(G(v, \overline{\mathbf{w}})-\frac{\partial \overline{\mathbf{w}}}{\partial t}\right) \overline{\mathbf{r}} \mathrm{d} \Omega \mathrm{d} t \\
& +\int_{0}^{T} \int_{\partial \Omega_{12}}\left(\eta \cdot \bar{\sigma}_{e} \nabla u-I_{e}\right) p \mathrm{~d} \Omega \mathrm{d} t
\end{aligned}
$$


where the initial and boundary conditions are kept as explicit constraints. The first order optimality system is obtained by formally setting the partial derivatives of $\mathcal{L}$ equal to 0 . After taking the derivative of $\mathcal{L}$ w.r.t. the state variables $u, v$ and $\overline{\mathbf{w}}$ the following adjoint equations for $p, q$ and $\overline{\mathbf{r}}$ are obtained.

Adjoint equations

$$
\begin{aligned}
0 & =\nabla \cdot\left(\bar{\sigma}_{i}+\bar{\sigma}_{e}\right) \nabla p+\nabla \cdot \bar{\sigma}_{i} \nabla q \quad \text { in } Q \\
\frac{\partial q}{\partial t} & =-\nabla \cdot \bar{\sigma}_{i} \nabla p-\nabla \cdot \bar{\sigma}_{i} \nabla q+I_{v}(v, \overline{\mathbf{w}}) q+G_{v}(v, \overline{\mathbf{w}}) \overline{\mathbf{r}} \text { in } Q \\
\frac{\partial r}{\partial t} & =-G_{\overline{\mathbf{w}}}(v, \overline{\mathbf{w}}) \overline{\mathbf{r}}+I_{\overline{\mathbf{w}}}(v, \overline{\mathbf{w}}) q \quad \text { in } Q,
\end{aligned}
$$

where the subscripts $v, \overline{\mathbf{w}}$ denote partial derivatives. Moreover, the terminal conditions are defined as follows.

$$
q(T)=0, \overline{\mathbf{r}}(T)=0
$$

The boundary conditions for the adjoint states are

$$
\begin{aligned}
\eta \cdot\left(\bar{\sigma}_{i} \nabla q+\bar{\sigma}_{i} \nabla p\right) & =0 & & \text { on } \Sigma, \\
\eta \cdot \bar{\sigma}_{e} \nabla p & =0 & & \text { on } \Sigma,
\end{aligned}
$$

and the compatibility condition for the adjoint variable $\int_{\Omega} p(t) d x=0$, for a.e. $t \in$ $(0, T)$. Moreover, for any optimal control $I_{e}^{*}$ the following variational inequality must be satisfied:

$$
\int_{0}^{T} \int_{\partial \Omega_{12}}\left(\alpha I_{e}^{*}+Q p\right)\left(I_{e}-I_{e}^{*}\right) d s d t \geq 0, \text { for all } I_{e} \in U
$$

where $(Q p)(t)=p(t)-\frac{1}{\partial \Omega_{12} \mid} \int_{\partial \Omega_{12}} p(t, s) d s$ on $\partial \Omega_{12}$. Here one needs a special numerical treatment to solve the Eq. (14) along with the boundary conditions Eqs. (17) and (18). In this regard, we adopted the stabilized finite element method to resolve this issue which is explained in Section 4.

\section{Numerical discretization}

The space and time discretizations to solve the partial differential equations in the optimality system is briefly explained. A piecewise linear finite element method is used for the spatial discretization of the primal and dual equations and their temporal discretization is done by using linearly implicit Runge-Kutta methods. 


\section{Semi-discretization in space}

Here we give an overview of the spatial discretization of the primal problem by a finite element method based on the weak formulation. A weak solution triple $(u, v, \overline{\mathbf{w}})$ satisfies for a.e. $t \in(0, T)$ and for all $\varphi \in H^{1}(\Omega)$

$$
\begin{aligned}
0 & =\left\langle\nabla \cdot\left(\sigma_{i}+\sigma_{e}\right) \nabla u+\nabla \cdot \sigma_{i} \nabla v, \varphi\right\rangle, \\
\left\langle\frac{\partial v}{\partial t}, \varphi\right\rangle & =\left\langle\nabla \cdot \sigma_{i} \nabla v+\nabla \cdot \sigma_{i} \nabla u-I_{i o n}(v, \overline{\mathbf{w}})+I_{t r}, \varphi\right\rangle, \\
\left\langle\frac{\partial \overline{\mathbf{w}}}{\partial t}, \varphi\right\rangle & =\langle G(v, \overline{\mathbf{w}}), \varphi\rangle,
\end{aligned}
$$

together with initial and boundary conditions (7)-(10). Let $V_{h} \subset H^{1}(\Omega)$ be the finite dimensional subspace of piecewise linear basis functions. The approximate solutions $\mathbf{u}, \mathbf{v}$ and $\mathbf{w}$ are expressed in the form $\mathbf{u}(t)=\sum_{i=1}^{N} u_{i}(t) \omega_{i}, \mathbf{v}(t)=$ $\sum_{i=1}^{N} v_{i}(t) \omega_{i}$, and $\mathbf{w}(t)=\sum_{i=1}^{N} \overline{\mathbf{w}}_{i}(t) \boldsymbol{\omega}_{i}^{T}$, respectively, where $\left\{\omega_{i}\right\}_{i=1}^{N}$ denote the basis functions. The semi-discretization of the primal equations in space results in the differential algebraic system as follows:

$$
\begin{aligned}
\mathbf{A}_{\mathbf{i e}} \mathbf{u}+\mathbf{A}_{\mathbf{i}} \mathbf{v} & =\mathbf{I}_{e} \\
\mathbf{M} \frac{\partial \mathbf{v}}{\partial t} & =-\mathbf{A}_{\mathbf{i}} \mathbf{v}-\mathbf{A}_{\mathbf{i}} \mathbf{u}-\mathbf{I}_{i o n}(\mathbf{v}, \mathbf{w})+\mathbf{I}_{t r}, \\
\mathbf{M} \frac{\partial \mathbf{w}}{\partial t} & =\mathbf{G}(\mathbf{v}, \mathbf{w}),
\end{aligned}
$$

along with initial conditions for $\mathbf{v}$ and $\mathbf{w}$, where $\mathbf{A}_{\mathbf{i e}}=\left\{\left\langle\left(\sigma_{i}+\sigma_{e}\right) \nabla \omega_{i}, \nabla \omega_{j}\right\rangle\right\}_{i, j=1}^{N}$ and $\mathbf{A}_{\mathbf{i}}=\left\{\left\langle\sigma_{i} \nabla \omega_{i}, \nabla \omega_{j}\right\rangle\right\}_{i, j=1}^{N}$ are the stiffness matrices, $\mathbf{M}=\left\{\left\langle\omega_{i}, \omega_{j}\right\rangle\right\}_{i, j=1}^{N}$ is the mass matrix. The vectors $\mathbf{I}_{e}, \mathbf{I}_{i t r}$ are defined by $\mathbf{I}_{e}=\left\{\left\langle\left(\chi_{\partial \Omega_{1}} I_{e}-\chi_{\partial \Omega_{2}} I_{e}\right), \omega_{j}\right\rangle\right\}_{j=1}^{N_{\partial \Omega}}$ and $\mathbf{I}_{t r}=\left\{\left\langle I_{t r}, \omega_{j}\right\rangle\right\}_{j=1}^{N}$, respectively. The expression for $\mathbf{I}_{f i}(\mathbf{v}, \mathbf{w})$ is defined by

$$
\mathbf{I}_{i o n}(\mathbf{v}, \mathbf{w})=\left(\mathbf{I}_{i o n}\right)\left(\sum_{i=0}^{N} v_{i} \omega_{i}, \sum_{i=0}^{N} \overline{\mathbf{w}}_{i} \boldsymbol{\omega}_{i}^{T}\right),
$$

In our computations we approached the linear system of Eqs. (25) and (26) is solved as coupled system. Then the discretized system is set up as follows:

$$
\left(\begin{array}{cc}
\mathbf{M} & 0 \\
0 & \mathbf{M}
\end{array}\right)\left(\begin{array}{c}
\frac{\partial \mathbf{v}}{\partial t} \\
\frac{\partial \mathbf{w}}{\partial t}
\end{array}\right)=\left(\begin{array}{c}
V(\mathbf{u}, \mathbf{v}, \mathbf{w}) \\
G(\mathbf{v}, \mathbf{w})
\end{array}\right)
$$

where $V(\mathbf{u}, \mathbf{v}, \mathbf{w})$ represents the right hand side of Eq. (25). A consistent spatial discretization is used for the dual equations. 


\section{Time discretization}

The semi-discretization of the Eq. (28) and also the adjoint equation after space discretization can be expressed in the following general form,

$$
\mathcal{M} \frac{\partial \mathrm{x}}{\partial t}=\mathcal{F}(\mathrm{x}), \quad \mathrm{x}\left(t^{0}\right)=\mathrm{x}^{0} .
$$

To solve (29), we introduce discrete steps:

$$
0=t^{0}, t^{1}, \ldots, t^{n}=T
$$

which are not necessarily equidistant. For the time discretization we employ linearly implicit Runge-Kutta, Rosenbrock, methods to solve the Eq. (29). These belong to a large class of methods which try to avoid the nonlinear system and replace it by a sequence of linear ones. Here we applied a second order Rosenbrock method called ROS3PL [16]. The construction of the Jacobian of the system is based on the exact derivatives of the right hand side vector $\mathcal{F}(\mathrm{x})$. We do not give the details here since they are well explained in [7, Section 3.2]. After the full discretization of the Eq (28) we obtain a system of linear algebraic equations. To solve this linear system we employed a BiCGSTAB method with ILU preconditioning. It is clear that the left hand side matrix of the algebraic system in all stages of the ROS3PL method is the same for each time step. In our computations, a LU decomposition is done once at the beginning of each time step and reused as ILU preconditioner in all internal stages at that time step. In this way, we can avoid some computational overhead during the linear solver phase. The solution of the discretized adjoint system, backward in time, is realized in a similar manner.

\section{Solution procedure}

Here we describe the solution procedure to solve the primal system in two steps. First, by utilizing the computed solutions $\mathbf{u}^{i}$ at time $t^{i}$ solve the discretized parabolic equation and the ODEs for $\mathbf{v}^{i+1}$ and $\mathbf{w}^{i+1}$ at time $t^{i+1}$ by applying the linearly implicit Runge-Kutta method. In the second step, using the available solution $\mathbf{v}^{i+1}$ at time $t^{i+1}$, solve the discretized elliptic system (24) for $\mathbf{u}^{i+1}$ by using a stabilized saddle point approach. The solution of the singular linear systems which arise after the full discretization of Eqs (2) and (14) are defined up to an additive constant. We mentioned earlier that we impose a zero mean condition to fix this constant. For the numerical realization of this condition we adopted a stabilized saddle point formulation from the work of Bochev and Lehoucq [4]. The discussion and implementation details of this technique for the current problem we refer to [7]. To solve this linear system we employed a BiCGSTAB [30] method with AMG preconditioner [3]. We can clearly observe that the left hand side matrix which arises from Eqs (2) and (14) is the same throughout the solution procedure. Only the right hand side vector changes at 
each time step during the primal or dual problem. It is well known that matrix hierarchy construction on coarser levels of AMG from a system matrix is as costly as solving the system $[3,24]$. In this regard, the constructed levels of the AMG hierarchy can be stored and this stored matrix hierarchy is re-used to solve the system for a given right-hand side throughout the primal and dual solve.

The complete optimality system is solved by the non-linear conjugate gradient (NCG) [19] using the Hager-Zhang [13] variant update. It is well known that gradient based algorithms exhibit slow convergence. In spite of that, due to their simple implementation they are the method of choice to numerically verify that the proposed optimal control approach is feasible. A line search procedure based on the Armijo rule with backtracking is used to determine the next update of the solution during the optimization iterations.

Parallelization can be used for numerical speeding. In fact, in our simulations we used the software package DUNE [1], which is a C++ template based programming environment for solving a general class of PDE's. The internal parallel Cartesian (called Yasp) grid in DUNE is used for parallel grid constructions. It was combined with non overlapping domain decomposition of the computational grid, see our previous article for more details [7].

\section{Numerical Results}

The computational domain is $\Omega=[0,5] \times[0,5] \subset \mathbb{R}^{2}$ of size $5 \star 5 \mathrm{~cm}^{2}$ and a $256 \times 256$ uniform quadrilateral spatial grid is used which consists of 65,536 elements and 66,049 nodes. Thus the spatial computation involved 264,196 dofs for one PDE solve. The computational domain setup with different components where the control acts on the left and right boundary is shown in Figure 1. On the discretized level, $\Omega_{o b s}$ is chosen as the domain which arises by removing the elements of the computational domain which are intersecting with the boundary $\Gamma_{12}$. The left boundary $\Gamma_{1}$ acts as a Anode and right boundary $\Gamma_{2}$ acts as a Cathode. Moreover, in this way the compatibility condition is satisfied for the elliptic solve in the primal equations which assure the existence of the solution. The parameters for characterization of cellular dynamics were taken from the original model in [17].

To obtain successful defibrillation the structural heterogeneity of the tissue plays a crucial role [28]. This can interfere with the spread of cardiac activation and contribute to initiation of spiral waves and wave break $[10,12]$. In our computational study, information on tissue heterogeneity is derived from a histological image, see left panel of Figure 2. First we converted the histological image to gray scale image, right panel of Figure 2. Subsequently, the generated gray scale image information was projected on to the computational mesh accordingly. We multiplied the conductivity tensor value by $10^{-3}$ where the white spots appear, so that the excitation wave front speed could have been reduced dramatically in those regions and the same conductivity tensor value was maintained at the 


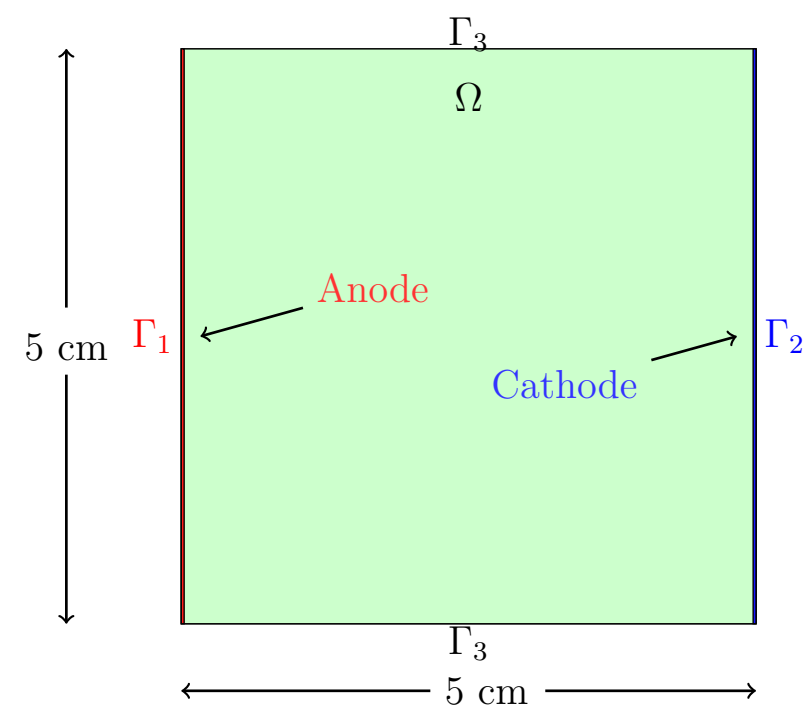

Figure 1: The computational domain setup with stimulation boundaries.

rest of the domain. In the presence of structural heterogeneity, the conduction velocities were 0.52 and $0.41 \mathrm{~m} / \mathrm{s}$ in the longitudinal and transverse direction, respectively.

In our computations, the stopping criteria for the optimization algorithm is based on the following conditions. Firstly,

$$
\left\|\nabla J\left(\mathbf{I}_{e}^{k}\right)\right\|_{L^{2}} \leq 10^{-5} \cdot\left|J\left(\mathbf{I}_{e}^{k}\right)\right| \text { or }\left|J\left(\mathbf{I}_{e}^{k}\right)-J\left(\mathbf{I}_{e}^{k-1}\right)\right| \leq 10^{-6}
$$

Moreover, the algorithm was terminated if this condition was not satisfied within a prescribed number of 150 iterations.

To induce the reentry wave we followed the standard $S 1-S 2$ stimulation protocol and the stimulus strength of $I_{t r}=50 \mu \mathrm{A} / \mathrm{cm}^{3}$ applied for the duration of $1 \mathrm{msec}$. The solution at $t=285 \mathrm{msec}$ was then chosen as the initial state for simulating the delivery of electrical shocks and the post-shock evolution following at the end of the shock. To create spiral wave phenotypes in the Luo-Rudy phase 1 membrane model, we altered the maximal conductance of the slow inward current $\left(G_{s i}=0.015\right)$. Apart from that, we changed the following two parameters in our simulations to $g_{N a}=16.0$ and $g_{K}=0.705$. The rest of the model parameters are taken directly from the original model [17]. The three temporal horizons are illustrated in Figure 3. During the shock, which constitutes the time period within which optimization takes place, the size of the time step was kept constant at $\Delta t=0.04 \mathrm{msec}$. During the pre- and post-shock phases adaptive time stepping features of the ROS3PL method were used to speed up computation.

The presented numerical results are done on a Linux cluster consisting of ten nodes where each node consists of 8 quad-core AMD Opteron processors 

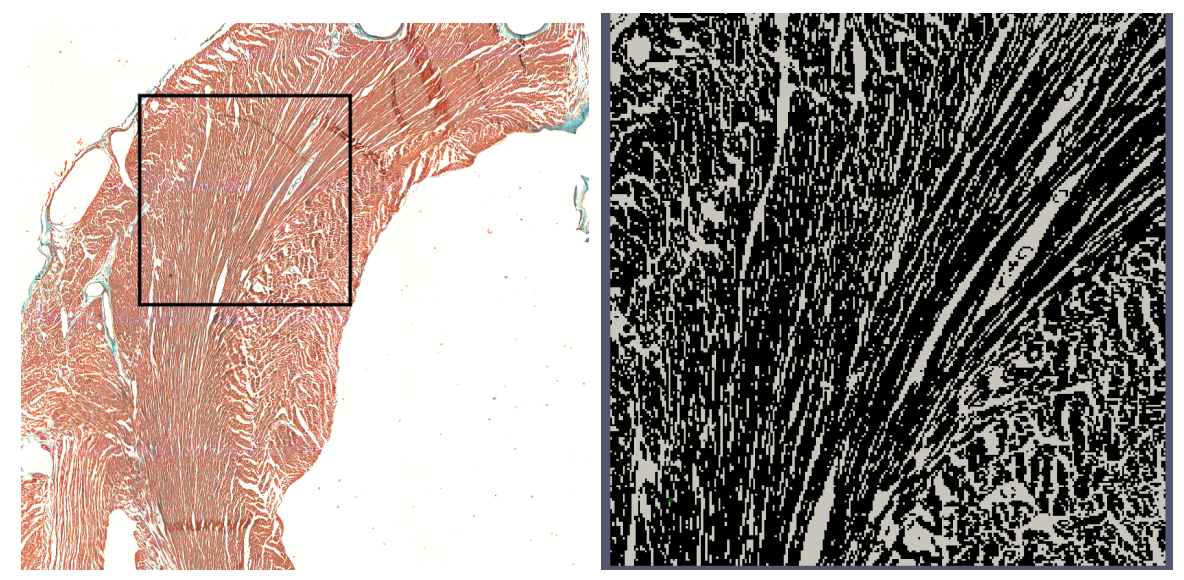

Figure 2: Histological image of the cardiac tissue at left panel. The selected region at the histological image converted to gray scale image at right panel for numerical computations [5].

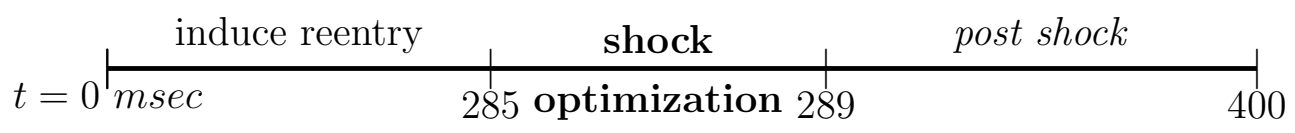

Figure 3: Different time horizons considered in the computations.

8356 clocked at $2.3 \mathrm{GHz}$ and equipped with 1TB RAM. All the presented results are based on the parallel NCG algorithm using 64 cores. The solution of the transmembrane voltage and the extracellular potential at time $t=285 \mathrm{msec}$ are depicted in Figure 4. We feed this solution as a initial solution for the optimization algorithm.
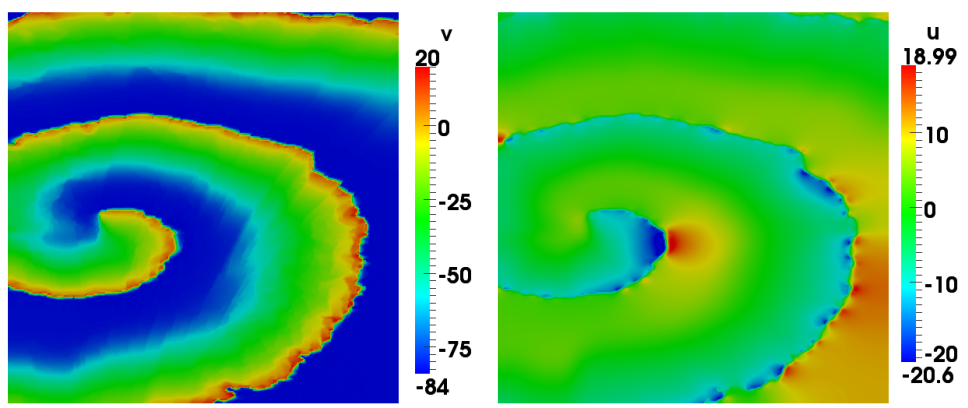

Figure 4: The solution of $v$ and $u$ at time $t=285 \mathrm{msec}$.

The solution for the transmembrane voltage $v$ in absence of any control is shown in Figure 5 at different instances of time to verify that the reentry is sustained for a sufficiently long time. 


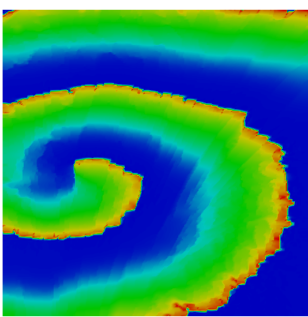

(a) $\mathrm{t}=289.0 \mathrm{msec}$

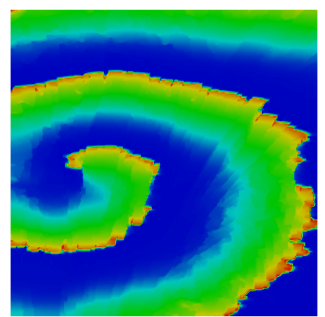

(b) $\mathrm{t}=294.5 \mathrm{msec}$

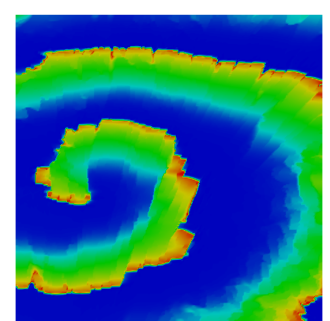

(c) $\mathrm{t}=318.5 \mathrm{msec}$

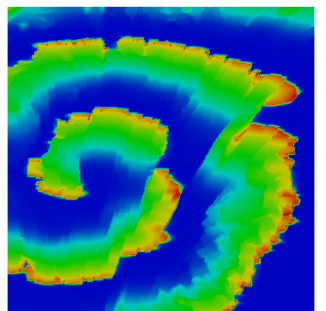

(d) $\mathrm{t}=380.5 \mathrm{msec}$

Figure 5: 2D visualization of uncontrolled solution $(v)$ at different times of simulation.

\subsection{Termination of reentrant waves}

In the computational domain, the anode is modeled as a Neumann boundary along the surface $\Gamma_{1}$ and the cathode along the surface $\Gamma_{2}$ of the computational domain as shown Figure 1. The desired trajectory of the transmembrane potential $\left(v_{d}\right)$ plays a crucial role to achieve successful defibrillation in post shock simulations. Indeed, choosing the right desired trajectory is not a trivial task. In our computations, this is obtained by solving once the primal problem using a prescribed time course of a stimulation current, $I_{e}(t)=10 \mathrm{~mA} / \mathrm{cm}^{3}$. The presented numerical results are based on fixing $\alpha_{1}=0.0005$ and varying the weight of the control parameter value $\alpha_{2}$ in the computations. The optimization algorithm constructs the best optimal control trajectory of the extracellular current while keeping the total current low, due to the appearance of term $\int I_{e}^{2}(t) \mathrm{d} t$ in cost functional.

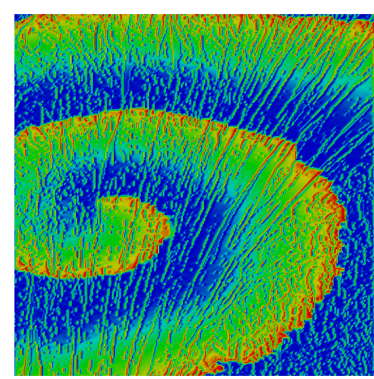

(a) $\mathrm{t}=285.2 \mathrm{msec}$

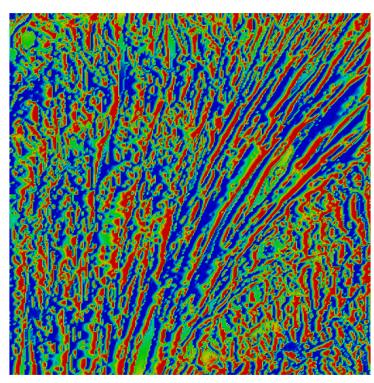

(b) $\mathrm{t}=287.0 \mathrm{msec}$

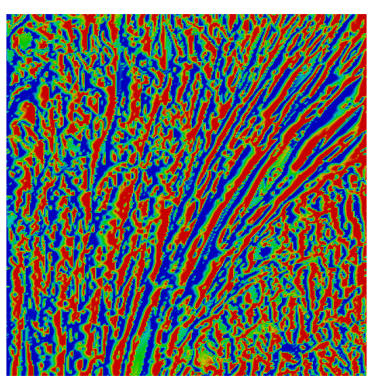

(c) $\mathrm{t}=289.0 \mathrm{msec}$

Figure 6: 2D visualization of optimal state solution $(v)$ at different times of simulation.

The 2D spatial representation of the optimized transmembrane voltage is shown in Figure 6 at different time instances. We can observe that during the shock period, at time $t=289 \mathrm{msec}$, a sufficiently large portion of the computational domain is depolarized. The applied external stimulus produces a large number of virtual electrodes at the microscopic size scale. After the break of the shock the virtual cathodes depolarize the virtual anodes quickly. Thus the 
entire domain is depolarized quickly which effectively blocks any further wave propagation or re-initiation of reentry, see the first panel in Figure 7, and eventually returns to the resting state at $381.43 \mathrm{msec}$, see the last panel of Figure 7. Compared to the optimal control of phenomenological FHN model [7] the optimal control of Luo-Rudy model is more complex in terms of gating dynamics and computational cost. We observed that the computational cost increases approximately a factor of 10 for the solution of the primal system. The primal solve during the first iteration of the optimization algorithm took 182.64 seconds on 64 cores. Here $6 \%$ of the CPU time is used for the solution of elliptic system, $15 \%$ for the left hand side matrix assembly of the coupled system and $70 \%$ for solving it. Solving the adjoint system took approximately the same CPU time. The CPU time for the complete optimization algorithm is 25.11 hours, while the post shock simulation took 31.60 minutes.

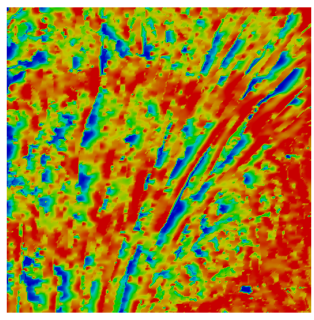

(a) $\mathrm{t}=293.65 \mathrm{msec}$

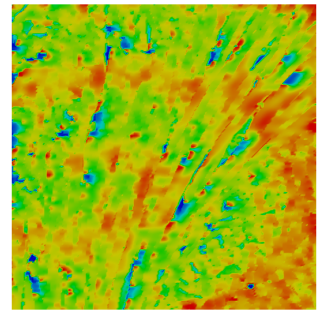

(b) $\mathrm{t}=297.43 \mathrm{msec}$

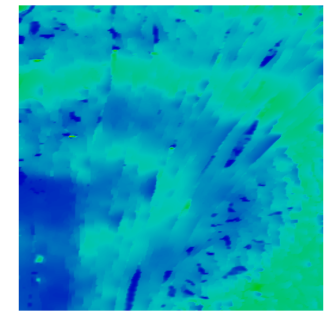

(c) $\mathrm{t}=317.43 \mathrm{msec}$

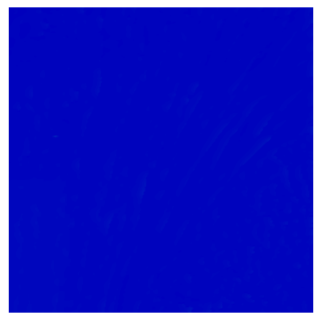

(d) $\mathrm{t}=381.43 \mathrm{msec}$

Figure 7: 2D visualization of controlled solution $(v)$ at different times of post shock simulation.

The norm of the gradient as a function of the NCG iterations for different regularization parameter values is shown in the left panel of Figure 8. For all parameter values the norm of the gradient decreases much more rapidly at the beginning than towards the end and they all approach to zero. In all cases, the optimization algorithm is terminated after 150 iterations due to not much progress in the gradient and the minimizational value. In middle panel of Figure 8 the cost functional value is shown. Here we can observe that the minimizational value at the end of the optimization is different for different regularization parameter values. The corresponding optimal value of the extracellular current is shown in the right panel of the Figure 8 where we can observe that the regularization parameter value $\alpha_{2}=0.05$ attained better optimal current compare to other parameter values. In all cases we observed that a successful defibrillation is achieved. Moreover, we observed that for value $\alpha_{2} \geq 0.05$ the termination of reentry wave is not possible. The total current required to construct the desired trajectory is $40 \mathrm{~mA} \mathrm{~ms} / \mathrm{cm}^{3}$. The total current obtained using the optimization is $35.5239 \mathrm{~mA} \mathrm{~ms} / \mathrm{cm}^{3}$ for $\alpha_{2}=0.05,38.3731 \mathrm{~mA} \mathrm{~ms} / \mathrm{cm}^{3}$ for $\alpha_{2}=0.01$ and 38.7905 $\mathrm{mA} \mathrm{ms} / \mathrm{cm}^{3}$ for $\alpha_{2}=0.001$. Thus the optimal control approach automatically constructs a current stimulus which leads to a successful defibrillation utilizing less total current. 

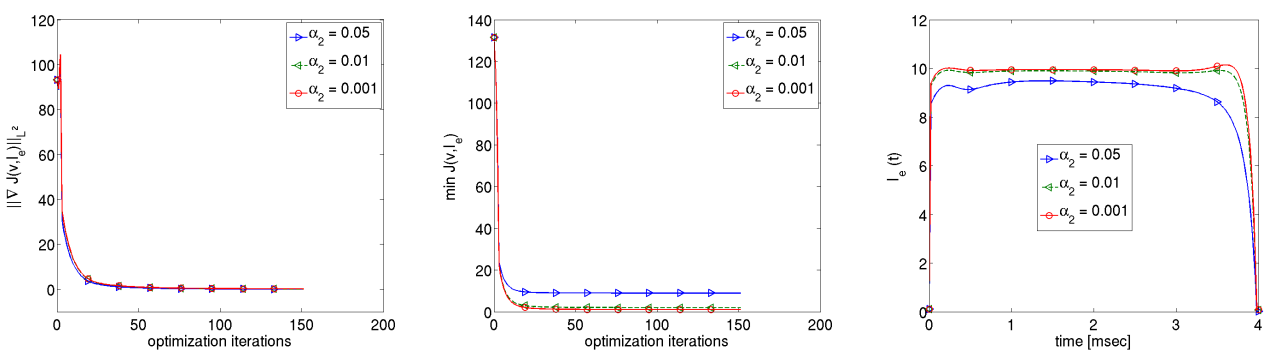

Figure 8: The gradient and minimum value of the cost functional and the optimal control values are shown on the left, middle and right respectively for different regularization parameter values $\alpha_{2}$.

\section{Conclusion}

The optimal control approach for the termination of reentrant waves in cardiac electrophysiology based on the bidomain model was discussed. The Luo-Rudy phase 1 membrane model was considered which represents excitability and refractoriness in a bio-physically more detailed as compared to the Fitz-Hugh-Nagumo model used in our previous studies [9]. Here the parabolic equation and the ODEs were solved on as a coupled system. This allowed us to use a fixed time step for primal and adjoint throughout the iteration of the optimization algorithm. This was not the case when decoupling the system.

The numerical results show that the optimal control strategy leads to successful defibrillation by designing optimal pulse waveforms which drive fibrillating cardiac tissue into a desired state with lower energy requirements when compared to ad-hoc strategies. Many important questions remain, including, for example, the problem of optimal actuator placement, and robustness of the control schemes. Furthermore, it is important to study the applicability of such optimal control techniques on cardiac tissue surrounded by a bath volume where the control acts on the surface of bath domain in order to be comparable with available experimental results.

\section{Acknowledgment}

The authors gratefully acknowledge the Austrian Science Foundation (FWF) for financial support under SFB 032, "Mathematical Optimization and Applications in Biomedical Sciences" and the Austrian Academy of Sciences (OÄW).

\section{References}

[1] P. Bastian, M. Blatt, A. Dedner, C. Engwer, R. Klöfkorn, R. Kornhuber, M. Ohlberger, and O. Sander. A generic grid interface for parallel and adaptive scientific computing. Part II: implementation and tests in DUNE. Computing, 82(2):121-138, July 2008. 
[2] G. W. Beeler and H. Reuter. Reconstruction of the action potential of ventricular myocardial fibres. J Physiol, 268(1):177-210, Jun 1977.

[3] M. Blatt. A parallel algebraic multigrid method for elliptic problems with highly discontinuous coefficients. $\mathrm{PhD}$ thesis, Ruprechts-Karls-Universität Heidelberg, 2010.

[4] P. Bochev and R. B. Lehoucq. On the finite element solution of the pure neumann problem. SIAM Rev., 47:50-66, January 2005.

[5] F. Campos, T. Wiener, A. Prassl, R. dos Santos, D. Sanchez-Quintana, H. Ahammer, G. Plank, and E. Hofer. Electroanatomical characterization of atrial microfibrosis in a histologically detailed computer model. Biomedical Engineering, IEEE Transactions on, 60(8):2339-2349, 2013.

[6] N. Chamakuri and K. Karl. Higher order optimization and adaptive numerical solution for optimal control of monodomain equations in cardiac electrophysiology. Appl. Numer. Math., 61:53-65, 2011.

[7] N. Chamakuri, K. Kunisch, and G. Plank. Optimal control approach to termination of re-entry waves in cardiac electrophysiology. Journal of Mathematical Biology, 67(2):1-30. 10.1007/s00285-012-0557-2.

[8] N. Chamakuri, K. Kunisch, and G. Plank. Numerical solution for optimal control of the reaction-diffusion equations in cardiac electrophysiology. Computational Optimization and Applications, 49:149-178, 2011. 10.1007/s10589-009-9280-3.

[9] N. Chamakuri, K. Kunisch, and G. Plank. On boundary stimulation and optimal boundary control of the bidomain equations. Mathematical Biosciences, 245(2):206 - 215, 2013.

[10] J. M. T. de Bakker and H. M. V. van Rijen. Continuous and discontinuous propagation in heart muscle. J Cardiovasc Electrophysiol, 17(5):567-573, May 2006.

[11] I. R. Efimov, R. A. Gray, and B. J. Roth. Virtual electrodes and deexcitation: new insights into fibrillation induction and defibrillation. J Cardiovasc Electrophysiol, 11(3):339-353, Mar 2000.

[12] Z. J. Engelman, M. L. Trew, and B. H. Smaill. Structural heterogeneity alone is a sufficient substrate for dynamic instability and altered restitution. Circulation: Arrhythmia and Electrophysiology, 3(2):195-203, 2010.

[13] W. Hager and H. Zhang. A new conjugate gradient method with guaranteed descent and an efficient line search. SIAM Journal on Optimization, 16(1):170-192, 2005. 
[14] C. S. Henriquez. Simulating the electrical behavior of cardiac tissue using the bidomain model. Crit. Rev. Biomed. Eng., 21:1 77, 1993.

[15] V. Henson and U. Yang. BoomerAMG: a Parallel Algebraic Multigrid Solver and Preconditioner. Applied Numerical Mathematics, 41:155-177, 2002.

[16] J. Lang and D. Teleaga. Towards a fully space-time adaptive FEM for magnetoquasistatics. IEEE Transactions on Magnetics, 44(6):1238-1241, 2008 .

[17] C. Luo and Y. Rudy. A model of the ventricular cardiac action potential: Depolarization, repolarization, and their interaction. Circ. Res., 68:15011526, 1991.

[18] S. A. Niederer, E. Kerfoot, A. P. Benson, M. O. Bernabeu, O. Bernus, C. Bradley, E. M. Cherry, R. Clayton, F. H. Fenton, A. Garny, E. Heidenreich, S. Land, M. Maleckar, P. Pathmanathan, G. Plank, J. F. Rodrguez, I. Roy, F. B. Sachse, G. Seemann, O. Skavhaug, and N. P. Smith. Verification of cardiac tissue electrophysiology simulators using an n-version benchmark. Philos Trans A Math Phys Eng Sci, 369(1954):4331-4351, Nov 2011.

[19] J. Nocedal and S. J. Wright. Numerical Optimization. Springer Verlag, New York, second edition edition, 2006.

[20] G. Plank, M. Liebmann, R. W. dos Santos, E. Vigmond, and G. Haase. Algebraic multigrid preconditioner for the cardiac bidomain model. IEEE Trans Biomed Eng., 54(4):585-596, 2007.

[21] G. Plank, A. Prassl, E. Hofer, and N. A. Trayanova. Evaluating intramural virtual electrodes in the myocardial wedge preparation: simulations of experimental conditions. Biophys J, 94(5):1904-1915, Mar 2008.

[22] R. Plonsey. Bioelectric sources arising in excitable fibers (ALZA lecture). Ann Biomed Eng, 16(6):519-46, 1988.

[23] J. M. Rogers and A. D. McCulloch. A collocation-Galerkin finite element model of cardiac action potential propagation. IEEE Trans. Biomed. Eng., 41:743-757, 1994.

[24] R. Scheichl and E. Vainikko. Additive schwarz with aggregation-based coarsening for elliptic problems with highly variable coefficients. Computing, 80(4):319-343, 2007.

[25] N. G. Sepulveda, B. J. Roth, and J. P. Wikswo, Jr. Current injection into a two-dimensional anisotropic bidomain. Biophys J, 55(5):987-99, 1989. 
[26] E. Sobie, R. Susil, and L. Tung. A generalized activating function for predicting virtual electrodes in cardiac tissue. Biophys J, 73(3):1410-23, 1997.

[27] K. Stüben. A review of algebraic multigrid. J Comput Appl Math, 128(12):281 - 309, 2001.

[28] N. Trayanova, K. Skouibine, and F. Aguel. The role of cardiac tissue structure in defibrillation. CHAOS, 8(1):221-233, MAR 1998.

[29] L. Tung. A bi-domain model for describing ischemic myocardial DC potentials. PhD thesis, MIT, Cambridge, MA, 1978.

[30] H. A. van der Vorst. Bi-CGSTAB: A fast and smoothly converging variant of bi-cg for the solution of nonsymmetric linear systems. SIAM J. Sci. Stat. Comput., 13:631-644, 1994.

[31] M. Veneroni. Reactiondiffusion systems for the macroscopic bidomain model of the cardiac electric field. Nonlinear Analysis: Real World Applications, 10(2):849 - 868, 2009.

[32] E. J. Vigmond, R. Weber dos Santos, A. J. Prassl, M. Deo, and G. Plank. Solvers for the cardiac bidomain equations. Prog Biophys Mol Biol, 96(13):3-18, 2008. 